\title{
Identification of Both Shared and Specific Potential Molecular Mechanisms of ARVC and DCM Based on A Genome-Wide Microarray Dataset
}

\section{Tang Zhang}

The Second Affiliated Hospital of Guangxi Medical University

\section{Yao-Zong Guan}

Guangxi Medical University

Hao Liu ( $D$ liuhaomd@126.com )

The Second Affiliated Hospital of Guangxi Medical University https://orcid.org/0000-0002-4105-8474

\section{Research article}

Keywords: Arrhythmogenic right ventricular cardiomyopathy, dilated cardiomyopathy, arrhythmia, differentially expressed gene, molecular mechanism

Posted Date: July 27th, 2020

DOl: https://doi.org/10.21203/rs.3.rs-47567/v1

License: (c) (i) This work is licensed under a Creative Commons Attribution 4.0 International License. Read Full License 


\section{Abstract}

Background囚The study aimed to detect the shared differentially expressed genes (DEGs) and specific DEGs of arrhythmogenic right ventricular cardiomyopathy (ARVC) and dilated cardiomyopathy (DCM) as well as their pathways.

Methods: The GSE29819 dataset was examined for the DEGs of ARVC vs. non-failing transplant donor hearts (NF), DCM vs. NF, and ARVC vs. DCM based on 8 patients with ARVC, 7 patients with DCM, and 4 non-failing transplant donor hearts that were never actually transplanted. The shared DEGs and specific DEGs were screened out using a Venn diagram. The Kyoto Encyclopedia of Genes and Genomes (KEGG) pathway enrichment, Gene Ontology (GO) annotation, and protein-protein interaction (PPI) of the DEGs were determined using online analytical tools. Then, the modules and hub genes were identified using Cytoscape software.

Results: A total of 684 shared DEGs of ARVC vs. NF and DCM vs. NF, 1371 specific DEGs of ARVC vs. NF, and 1075 specific DEGs of DCM vs. NF were identified. The shared DEGs were enriched in 63 biological processes (BP), 11 molecular functions (MF), 10 cellular components (CC), and 25 KEGG pathways. The DEGs of ARVC vs. DCM were enriched in $71 \mathrm{BPs}, 19 \mathrm{MFs}, 14 \mathrm{CCs}$, and $26 \mathrm{KEGG}$ pathways. A PPI network with 187 nodes, 700 edges, and 2 modules, and another PPI network with 575 nodes, 2834 edges, and 7 modules were constructed based on the shared and specific DEGs, respectively. The top ten hub genes CCR3, CCR5, CXCL2, CXCL 10, CXCR4, FPR1, APLNR, PENK, BDKRB2, GRM8, and RPS8, PRS3A, PRS12, RPS14, RPS21, RPL 14, RPL18A, RPL21, RPL31 were identified for the shared and specific PPI networks, respectively.

Conclusions: Our findings may help further the understanding of both shared and specific potential molecular mechanisms of ARVC and DCM.

\section{Background}

Arrhythmogenic right ventricular cardiomyopathy (ARVC) and dilated cardiomyopathy (DCM) are two types of cardiomyopathy. Both can lead to arrhythmia, heart failure (HF), and sudden cardiac death (SCD), and both have been found to be associated with genetic factors [1]. They rank as the second and fifth major causes of non-ischemic heart disease in SCD [2], respectively. It is estimated that there are 38 million cases of HF worldwide, and the prevalence in the adult population in developed countries is 1$2 \%[3,4]$. For SCD, in the United States alone, the incidence is estimated to be 60 per 100,000 individuals [5]. This has resulted in a heavy economic burden to countries and individuals, and has caused the death of many loved ones. At present, symptomatic therapies, including anti-arrhythmia treatment, anti-heart failure treatment, and ICD/CRT/CRT-D implantation, are the mainstays of therapy for ARVC and DCM, but treatments for the actual etiology are relatively rare. Although the clinical manifestations of ARVC and DCM are similar, it has not yet been clearly demonstrated whether ARVC and DCM share common genes and pathways. 
Previously called arrhythmogenic right ventricular dysplasia, ARVC is an autosomal dominant inherited cardiomyopathy that manifests as the replacement of the right ventricular myocardium with fatty and fibrous tissue, and its prevalence is estimated to be 1:5000 [6]. The causative genes accounting for $50 \%$ of ARVC have been identified, including PKP2 (Plakophilin-2) [7], DSP (Desmoplakin) [8], DSG2 (Desmoglein-2) [9], and JUP(Junctional-plakophilin) [10]. It is a complex disease involving multiple genes; although the unique manifestations of electrocardiogram (ECG), cardiac ultrasound, and cardiac magnetic resonance imaging (MRI) have greatly improved the sensitivity and specificity of ARVC diagnosis [11], little is still known about many of the potential pathogenic genes and regulatory pathways of this disease.

DCM is mainly characterized by ventricular enlargement and a decrease in the myocardial contraction force, with a prevalence of about 1:250 [12]. Some studies point out that both familial DCM and a fraction of non-familial DCM have a genetic basis $[13,14]$. To date, a portion of the key pathogenic genes of DCM have been identified, such as TTN [15], RBM20 [16], LMNA [17], and SCN5A [18]. Among them, a rare $T T N$ variant accounts for $15-25 \%$ of DCM while $R B M 20$ accounts for $1-3 \%$. In addition, $6 \%$ of DCM cases is caused by rare variants of $\angle M N A$, and these mutations are associated with the highest risk of SCD. Diverse cellular proteins and pathologic mechanisms are involved in the development of DCM, such as the cytoskeleton, desmosomes, and ion channels [1]. However, a number of potentially associated genes and pathways may not have been identified yet.

In this study, we used a genome-wide microarray dataset in human ventricular tissues to identify DEGs between ARVC and non-failing transplant donor hearts that were not transplanted due to technical issues, DCM and these same non-failing transplant donor hearts, and ARVC and DCM. Based on these DEGs, GO and KEGG pathways were analyzed, and then we used Cytoscape software to construct PPI networks and identify the hub genes, aiming to detect the shared and unique molecular mechanisms in ARVC and DCM.

\section{Methods}

\section{Affymetrix microarray data}

The GSE29819 gene expression dataset was retrieved from the Human Genome U133 Plus 2.0 Array platform (Affymetrix; Thermo Fisher Scientific, Inc., Waltham, MA, USA) from the GEO database (https://www.ncbi.nlm.nih.gov/geo/query/acc.cgi?acc=GSE29819). The GSE29819 dataset is comprised of 19 pairs of ventricular myocardial tissues, including 12 samples from 6 patients with ARVC (6 LV tissues vs. 6 RV tissues), 14 samples from 7 patients with DCM (7 LV tissues vs. 7 RV tissues), and 12 samples from non-failing (NF) donor hearts that could not be transplanted for technical reasons (6 LV tissues vs. 6 RV tissues).

\section{Identification Of Differentially Expressed Genes}


The samples from the GSE29819 dataset were divided into three groups: an ARVC group (12 samples), a DCM group (14 samples), and a non-failing group (12 samples). Afterwards, the DEGs of the ARVC group vs. the NF group, the DCM group vs. the NF group, and the ARVC group vs. the DCM group were identified using an online analytical tool called GEO2R. GEO2R is used for conducting comparisons on raw data based on the GEO-query and limma $\mathrm{R}$ package. The results were extracted to a file, and the screening criteria were as follows: $P<0.05$, and $\mid$ log fold-changel $>1.0$. The heat maps and volcano plots of DEGs were created using an R package. Then, the shared DEGs and the specific DEGs of the ARVC group vs. the NF group and the DCM group vs. the NF group were identified using the online analytical tool Draw Venn Diagram (http://bioinformatics.psb.ugent.be/webtools/Venn/).

\section{GO and KEGG pathway analyses and integration of the PPI network}

GO and KEGG pathway analyses of the DEGs were conducted separately on the Database for Annotation, Visualization and Integrated Discovery (DAVID) (version 6.8). Statistical significance was set at $P$-value< 0.05 . The results of the GO and KEGG pathway analyses were visualized by the R-ggplot2 package (version 3.5.3). Interactions among the shared DEGs and the DEGs of the ARVC group vs. the DCM group were evaluated using the STRING database (version 10.5) with a combined score of $>0.9$, and the results were downloaded in TSV format for visualization using the Cytoscape plugin cytoHubba (version 0.1). Then, the Cytoscape plugin Molecular Complex Detection (MCODE; version 1.31) was used to identify molecular modules of the PPI network, with a screening criterion of Degree cut-off $>10$ and K-Core $>10$, and cytoHubba was used to identify the top 10 hub genes according to maximal clique centrality (MCC) rank.

\section{Results}

\section{Identification of differential expression genes}

A total of 2055 DEGs (826 up-regulated and 1229 down-regulated) of the ARVC group vs. NF group, 1759 DEGs (772 up-regulated and 987 down-regulated) of the DCM group vs. NF group, and 1658 DEGs (1264 up-regulated and 788 down-regulated) of ARVC group vs. DCM group were identified, respectively. After screening with the Venn diagram, a total of 1371 specific DEGs of the ARVC group vs. NF group, 1075 specific DEGs of the DCM group vs. NF group, and 684 shared DEGs of the ARVC and DCM groups were determined (Fig. 1). Additionally, we screened out the top 100 DEGs in ascending $P$-value order to draw the heat map and volcano plots (Fig. 2).

\section{Go And Kegg Pathway Enrichment Analysis}

Among the shared DEGs, 63 biological processes (BP), 11 molecular functions (MF), and 10 cellular components (CC) were revealed by GO function clustering, and $25 \mathrm{KEGG}$ pathway enrichments were identified. Of the DEGs of the ARVC group vs. DCM group, $71 \mathrm{BPs}, 19 \mathrm{MFs}$, and $14 \mathrm{CCs}$, and $26 \mathrm{KEGG}$ pathway enrichments were revealed. As for the specific DEGs of the ARVC group vs. NF group, and the 
DCM group vs. NF group, 64 BPs, 19 MFs, 14 CCs, and 12 KEGG pathway enrichments, and 39 BPs, 16 MFs, $17 \mathrm{CCs}$, and $2 \mathrm{KEGG}$ pathway enrichments were revealed, respectively. The entire results of GO are shown in Supplementary Table 1, Supplementary Table 2, and Supplementary Table 3. The results of KEGG pathways and the top 10 BPs, MFs, and CCs of GO based on different DEGs were selected for visualization (Fig. 3). The shared pathways of the ARVC vs. NF and the DCM vs. NF, as well as ARVC vs. DCM, are shown in Fig. 4.

\section{Ppi Network Construction And Hub Gene Identification}

One PPI network with 187 nodes and 700 edges and another PPI network with 575 nodes and 2834 edges were constructed to detect the interactions among the shared DEGs and the interactions among DEGs of AVRC vs. DCM with a combined score $>0.9$ (Fig. $5 \mathrm{~A}$ and Fig. 6A). After analysis with Degree cut-off $>10$ and K-Core > 10, 2 modules (Fig. 5B and Fig. 5C) and 5 modules (Fig. 6B-F) were identified, respectively. Finally, the top 10 hub genes of the two PPI networks were screened out according to the rank of MCC (Fig. 5D and Fig. 6G).

\section{Discussion}

\section{The shared pathways of ARVC and DCM compared to HF}

The shared DEGs were enriched on the 25 pathways, as shown in Figure 2. Cytokines play important role in regulation of immune function and are associated with the occurrence and development of a large number of human diseases, and many cardiovascular diseases have been shown to be associated with uncontrolled cytokines [19]. The activity of intracellular Janus kinases (JAKs) is associated with the assembly of the cytokine-receptor complex in the classic cytokine signaling pathway [20]. JAKs phosphorylate and activate the signal transducer and activator of transcription (STAT), which subsequently modulates gene expression [21, 22]. IL-2, IL-4, and IL- 6 are the most common types of cytokine; among them, IL-6 first forms a dimer with IL-6R prior to binding its cognate receptor gp 130, which is constitutively associated with JAK family tyrosine (Tyr) kinases and can be phosphorylated by JAKs. In addition, IL-6 also activates phosphatidylinositol 3-kinase (PI3K) pathways and extracellular signal-regulated kinase (ERK) $1 / 2$, following recruitment of SH2-containing protein Tyr phosphatase 2 to JAK-phosphorylated gp130 [23]. PI3Ka/Akt signaling leads to phosphorylation of $\mathrm{Na}_{\mathrm{v}} 1.5$ on a site that regulates its gating properties, thus suppressing persistent $I_{\mathrm{Na}}$. At some point, the decrease of $\mathrm{Na}_{\mathrm{v}} 1.5$ on the cell surface may result in a decrease in peak $I_{\mathrm{Na}}$ after the inhibition of PI3Ka, which could slow action potential conduction and further induce arrhythmia if large enough [24]. These factors interact with pathways, some of which, if unregulated or unbalanced, may induce arrhythmias.

Many previous reports have proposed theories related to ARVC and DCM, including immunity, apoptosis, and gene mutation-related theories $[25,26]$. FoxO activity is involved in immune response, apoptosis, oxidative stress, aging, and other biological processes, mainly regulated by the PI3K/Art signaling 
pathway [27]. Similarly, the $p 53$ signal pathway and $T N F$ signal pathway are important components of apoptosis pathways. Consistent with previous reports, our study found the FoxO signal pathway, the p53 signal pathway, and TNF signaling pathways among the shared genes. The results suggest that immunity and apoptosis may be the common pathogenesis of ARVC and DCM, and intervention based on cell immunity and apoptosis may have significance for the prevention and treatment of ARVC and DCM.

Electrical remodeling is an important mechanism of arrhythmia and heart failure. After the integrity of myocardial fibers is destroyed, the anisotropy of electrical activity increases, which promotes conduction disorders, inducing arrhythmias and heart failure. Shimizu H. et al [28] revealed that overload of $\mathrm{Ca}^{2+} \mathrm{can}^{2}$ destroy the integrity of myocardial fibers by activating a calcineurin-FoxO-MuRF1-proteasome signaling pathway. Mota R. et al[29] reported a novel link between atrogin-1-mediated regulation of FoxO1/3 activity, reduced collagen deposition, and fibrosis in the aged heart. Bagchi AK. et al[30] showed that under stress, IL-10-mediated toll-like receptor 4 (TLR4) signaling suppresses apoptosis as well as fibrosis, while TLR2 has the opposite effect. Similarly, the hypoxia-inducible factor-1 (HIF-1) signal pathway also takes part in the development of fibrosis [31]. In the present study, the FoxO signaling pathway, HIF-1 signaling pathway, and toll-like receptor signaling pathway were all discovered to be shared DEGs. Thus, pathway-based interventions may help preserve the integrity of primary myocardial fibers, improve electrical remodeling, and reduce the occurrence of heart failure in patients with ARVC or DCM.

\section{The specific pathways of ARVC compared to HF and DCM compared to HF}

Specific pathways of ARVC compared to HF were identified in our study. Among them, the ECM-receptor interaction had already been proven to be involved in the fibrosis of myocardium [32]. The rest of the pathways tended toward neuro-regulation (neuroactive ligand-receptor interaction, serotonergic synapse, dopaminergic synapse, Fc gamma R-mediated phagocytosis) and infection (Leishmaniasis and Staphylococcus aureus infection). By contrast, the specific pathways of DCM compared to HF were related to ATP-binding cassette $(A B C)$ transporters and glycerolipid metabolism. ABC transporters like $A B C A 1, A B C A 4$, and $A B C A 5$ are all expressed in human platelets, and they regulate platelet function [33]. The ATP-binding cassette transporter P-glycoprotein (ABCB1) may affect the bioavailability and elimination of digoxin, while ABCA8 and ABCA9 are indispensable components of the ATP-sensitive potassium $\left(K_{A T P}\right)$ channel [34]. Glucose and lipid metabolism is important for myocardial cells, and some research shows that metabolic disorders are a cause of chronic heart failure, and that several parameters are even biological indicators of prognosis [35]. According to our results, development of ARVC and DCM occur via their unique pathways, and these pathways may provide some evidence to support targeted intervention for the two diseases, respectively.

\section{The pathways of ARVC compared to DCM}

Compared to the pathways based on the shared DEGs, some of the pathways that are enriched in ARVC vs. $D C M$ are unique, including $A B C$ transporters, signaling pathways that regulate the pluripotency of 
stem cells, type II diabetes mellitus, fat digestion and absorption, bile secretion, complement and coagulation cascades, and inflammatory bowel disease (IBD). Type II diabetes mellitus, fat digestion and absorption, and bile secretion are correlated with glucose and lipid metabolism. As we mentioned above, metabolic syndrome is a risk factor for heart failure. Metabolic disorders such as those related to glucose, fat, or protein metabolism may contribute to heart failure. The complement system is a major element of immune response, and also plays an important role in the development of IBD [36]. Whether the complement system activated by inflammatory bowel disease has any effect on the development of ARVC or DCM needs further study.

\section{Hub genes of the PPI network}

As the Figure 5 and Figure 6 show, we identified the top 10 hub genes of the ARVC vs. NF and DCM vs. NF, and ARVC vs. DCM, respectively. These genes are involved with metabolism, inflammation, immune, cell apoptosis, or other critical biological processes. Proenkephalin (PENK), which is related to renal function, is a stable endogenous opioid biomarker and has been reported to be a prognostic indicator of heart failure [37]. What's more, it is also a modulator of IL-10 [38], a cytokine involved in inflammation and immune response, which also have important roles in cardiovascular disease. BDKRB2 is related to hypertension as a target of angiotensin II type 1 receptor signaling, and its polymorphism is related to the glucose metabolism $[39,40]$. Endothelial $A P L N R$ is critical for apelin signaling and its glucose-lowering effects [41]. CCR3 and CCR5 are chemokine receptors, and the former plays a pivotal role in leukocyte chemotaxis [42]. CXC chemokines regulate the recruitment of neutrophils via CXCR1 and CXCR2 in humans [43]. In addition, CXCL 10 has demonstrated a novel function in mediating monocyte production of pro-inflammatory cytokines [44]. Another gene, CXCR4, was postulated to mediate atherosclerosis and inflammation in a recent study [45]. These genes played a role in immunity and/or inflammation, which has been demonstrated to be related to the development of cardiovascular disease.

In the context of the hub genes of the PPI network based on ARVC vs. DCM, RPS3A was a key factor in modulating the brown fat-specific gene UCP-1 and carbon metabolic enzymes in EAT for preventing CAD [46]. CDK11p46 and RPS8 are associated with each other, and both are involved in cell apoptosis; similarly, over-expression of RPS14 can inhibit Rb phosphorylation and result in cell cycle arrest and senescence $[47,48]$. It is well known that diabetes and renal dysfunction can both affect cardiac function, but interestingly, RPS12 has been identified as a pathogenic gene of diabetic kidney disease by a genome-wide association study (GWAS) [49]. Finally, the mutations of RPL18A and RPL31 have been proven to be associated with Diamond-Blackfan anemia [50], which may decrease the blood supply to myocardial cells to some degree.

Although the function and the pathway of the above hub genes have been reported previously, they have not been verified before, as a large enough sample of myocardium tissues with ARVC and DCM is difficult to collect. Meanwhile, it can also be inferred that since the mechanisms of ARVC and DCM are regulated by multiple genes and multiple pathways, they require more comprehensive and targeted intervention. 


\section{Limitations}

There are some limitations to our study. Firstly, the samples from non-failing donors may differ from the normal population, which may limit the applicability of our results between patients with ARVC or DCM and normal population. Secondly, since genes interact with each other, we artificially screened the intersection of different groups of DEGs for further analysis and may have inadvertently excluded some genes with potential links, which may make the analysis of diseases one-sided to some degree. Thirdly, our study was only based on the GSE29819 dataset, and the results need to be validated with a further, more rigorous investigation and a large sample size .

\section{Conclusions}

In this study, genome-wide differentially expressed genes were used to identify the functions and mechanism of shared and specific genes of patients with ARVC and/or DCM compared to non-failing donor heart patients. Our findings may help to provide a better understanding of the functions and roles of these DEGs in ARVC and DCM and provide a reference for future treatment strategies. However, further studies are required to validate the role of these DEGs and pathways involved in these two diseases.

\section{Abbreviations}

GEO

Gene Expression Omnibus; GO:Gene Ontology annotation; KEGG:Kyoto Encyclopedia of Genes and Genomes pathway enrichment analyses; MCODE:Molecular Complex Detection; PPI:Protein-protein interaction; BP:Biological processes; CC:Cellular components; MF:Molecular functions; ARVC:Arrhythmogenic right ventricular cardiomyopathy; DCM:Dilated cardiomyopathy; HF:Heart failure; SCD:Sudden cardiac death; DAVID:Database for Annotation, Visualization and Integrated Discovery; DEGs:Differentially expressed genes

\section{Declarations}

\section{Acknowledgements}

Not applicable.

\section{Funding}

No funding was received.

\section{Availability of data and materials}

The datasets used and/or analyzed during the current study are available from the Gene Expression Omnibus repository (https://www.ncbi.nlm.nih.gov/geo/query/acc.cgi?acc=GSE29819). 


\section{Authors' contributions}

T.Z. and Y.-Z. G. conceived the study, participated in the design, performed the statistical analysis, and drafted the manuscript. H. L. conceived the study, participated in the design and helped to draft the manuscript. All authors read and approved the final manuscript.

\section{Ethics approval and consent to participate}

Not applicable.

\section{Consent for publication}

Not applicable.

\section{Competing interests}

The authors declare that they have no competing interests.

\section{References}

1. Wilcox JE, Hershberger RE. Genetic cardiomyopathies. Curr Opin Cardiol. 2018;33(3):354-62.

2. Kuriachan VP, Sumner GL, Mitchell LB. Sudden cardiac death. Curr Probl Cardiol. 2015;40(4):133200.

3. Braunwald E. The war against heart failure: the Lancet lecture. The Lancet. 2015;385(9970):812-24.

4. Mosterd A, Hoes AW. Clinical epidemiology of heart failure. Heart. 2007;93(9):1137-46.

5. Stecker EC, Reinier K, Marijon E, Narayanan K, Teodorescu C, Uy-Evanado A, Gunson K, Jui J, Chugh SS. Public health burden of sudden cardiac death in the United States. Circ Arrhythm Electrophysiol. 2014;7(2):212-7.

6. Peters S, Trummel M, Meyners W. Prevalence of right ventricular dysplasia-cardiomyopathy in a nonreferral hospital. Int J Cardiol. 2004;97(3):499-501.

7. Gerull B, Heuser A, Wichter T, Paul M, Basson CT, McDermott DA, Lerman BB, Markowitz SM, Ellinor $\mathrm{PT}$, MacRae CA, et al. Mutations in the desmosomal protein plakophilin-2 are common in arrhythmogenic right ventricular cardiomyopathy. Nat Genet. 2004;36(11):1162-4.

8. Rampazzo A, Nava A, Malacrida S, Beffagna G, Bauce B, Rossi V, Zimbello R, Simionati B, Basso C, Thiene G, et al. Mutation in Human Desmoplakin Domain Binding to Plakoglobin Causes a Dominant Form of Arrhythmogenic Right Ventricular Cardiomyopathy. The American Journal of Human Genetics. 2002;71(5):1200-6.

9. Pilichou K, Nava A, Basso C, Beffagna G, Bauce B, Lorenzon A, Frigo G, Vettori A, Valente M, Towbin $\mathrm{J}$, et al. Mutations in desmoglein-2 gene are associated with arrhythmogenic right ventricular cardiomyopathy. Circulation. 2006;113(9):1171-9. 
10. McKoy G, Protonotarios N, Crosby A, Tsatsopoulou A, Anastasakis A, Coonar A, Norman M, Baboonian C, Jeffery S, McKenna WJ. Identification of a deletion in plakoglobin in arrhythmogenic right ventricular cardiomyopathy with palmoplantar keratoderma and woolly hair (Naxos disease). The Lancet. 2000;355(9221):2119-24.

11. Gandjbakhch E, Redheuil A, Pousset F, Charron P, Frank R. Clinical Diagnosis, Imaging, and Genetics of Arrhythmogenic Right Ventricular Cardiomyopathy/Dysplasia: JACC State-of-the-Art Review. J Am Coll Cardiol. 2018;72(7):784-804.

12. Hershberger RE, Hedges DJ, Morales A. Dilated cardiomyopathy: the complexity of a diverse genetic architecture. Nat Rev Cardiol. 2013;10(9):531-47.

13. Hershberger RE, Norton N, Morales A, Li D, Siegfried JD, Gonzalez-Quintana J. Coding sequence rare variants identified in MYBPC3, MYH6, TPM1, TNNC1, and TNNI3 from 312 patients with familial or idiopathic dilated cardiomyopathy. Circ Cardiovasc Genet. 2010;3(2):155-61.

14. Haas J, Frese KS, Peil B, Kloos W, Keller A, Nietsch R, Feng Z, Muller S, Kayvanpour E, Vogel B, et al. Atlas of the clinical genetics of human dilated cardiomyopathy. Eur Heart J. 2015;36(18):1123-35a.

15. Herman DS, Lam L, Taylor MR, Wang L, Teekakirikul P, Christodoulou D, Conner L, DePalma SR, McDonough B, Sparks E, et al. Truncations of titin causing dilated cardiomyopathy. N Engl J Med. 2012;366(7):619-28.

16. Guo W, Schafer S, Greaser ML, Radke MH, Liss M, Govindarajan T, Maatz H, Schulz H, Li S, Parrish AM, et al. RBM20, a gene for hereditary cardiomyopathy, regulates titin splicing. Nat Med. 2012;18(5):766-73.

17. van Rijsingen IA, Arbustini E, Elliott PM, Mogensen J, Hermans-van Ast JF, van der Kooi AJ, van Tintelen JP, van den Berg MP, Pilotto A, Pasotti M, et al. Risk factors for malignant ventricular arrhythmias in lamin a/c mutation carriers a European cohort study. J Am Coll Cardiol. 2012;59(5):493-500.

18. McNair WP, Sinagra G, Taylor MR, Di Lenarda A, Ferguson DA, Salcedo EE, Slavov D, Zhu X, Caldwell $\mathrm{JH}$, Mestroni L. SCN5A mutations associate with arrhythmic dilated cardiomyopathy and commonly localize to the voltage-sensing mechanism. J Am Coll Cardiol. 2011;57(21):2160-8.

19. Spangler JB, Moraga I, Mendoza JL, Garcia KC. Insights into cytokine-receptor interactions from cytokine engineering. Annu Rev Immunol. 2015;33:139-67.

20. Ihle JN, Witthuhn BA, Quelle FW, Yamamoto K, Silvennoinen O. Signaling through the hematopoietic cytokine receptors. Annu Rev Immunol. 1995;13:369-98.

21. O'Shea JJ, Plenge R. JAK and STAT signaling molecules in immunoregulation and immune-mediated disease. Immunity. 2012;36(4):542-50.

22. Levy DE, Darnell JE Jr. Stats: transcriptional control and biological impact. Nature reviews Molecular cell biology. 2002;3(9):651-62.

23. Schaper F, Rose-John S. Interleukin-6: Biology, signaling and strategies of blockade. Cytokine Growth Factor Rev. 2015;26(5):475-87. 
24. Ballou LM, Lin RZ, Cohen IS. Control of Cardiac Repolarization by Phosphoinositide 3-Kinase Signaling to lon Channels. Circ Res. 2015;116(1):127-37.

25. Que D, Yang P, Song X, Liu L. Traditional vs. genetic pathogenesis of arrhythmogenic right ventricular cardiomyopathy. Europace. 2015;17(12):1770-6.

26. Kawai C, Matsumori A. Dilated cardiomyopathy update: infectious-immune theory revisited. Heart Fail Rev. 2013;18(6):703-14.

27. Ronnebaum SM, Patterson C. The FoxO family in cardiac function and dysfunction. Annu Rev Physiol. 2010;72:81-94.

28. Shimizu H, Langenbacher AD, Huang J, Wang K, Otto G, Geisler R, Wang Y, Chen JN. The CalcineurinFoxO-MuRF1 signaling pathway regulates myofibril integrity in cardiomyocytes. Elife. 2017;6:e27955.

29. Mota R, Parry TL, Yates CC, Qiang Z, Eaton SC, Mwiza JM, Tulasi D, Schisler JC, Patterson C, Zaglia T, et al. Increasing Cardiomyocyte Atrogin-1 Reduces Aging-Associated Fibrosis and Regulates Remodeling in Vivo. Am J Pathol. 2018;188(7):1676-92.

30. Bagchi AK, Akolkar G, Mandal S, Ayyappan P, Yang X, Singal PK. Toll-like receptor 2 dominance over Toll-like receptor 4 in stressful conditions for its detrimental role in the heart. Am J Physiol Heart Circ Physiol. 2017;312(6):H1238-H47.

31. Ruthenborg RJ, Ban JJ, Wazir A, Takeda N, Kim JW. Regulation of wound healing and fibrosis by hypoxia and hypoxia-inducible factor-1. Mol Cells. 2014;37(9):637-43.

32. Buttner P, Ueberham L, Shoemaker MB, Roden DM, Dinov B, Hindricks G, Bollmann A, Husser D. Identification of Central Regulators of Calcium Signaling and ECM-Receptor Interaction Genetically Associated With the Progression and Recurrence of Atrial Fibrillation. Front Genet. 2018;9:162.

33. Wang W, Buitrago L, Wang Y. ABC transporters in megakaryopoiesis and platelet activity. Thromb Res. 2017;156:126-33.

34. Solbach TF, Konig J, Fromm MF, Zolk O. ATP-binding cassette transporters in the heart. Trends Cardiovasc Med. 2006;16(1):7-15.

35. Contaifer D Jr, Buckley LF, Wohlford G, Kumar NG, Morriss JM, Ranasinghe AD, Carbone S, Canada JM, Trankle C, Abbate A, et al. Metabolic modulation predicts heart failure tests performance. PLoS One. 2019;14(6):e0218153.

36. Sina C, Kemper C, Derer S. The intestinal complement system in inflammatory bowel disease: Shaping intestinal barrier function. Semin Immunol. 2018;37:66-73.

37. Kanagala P, Squire IB, Jones DJL, Cao TH, Chan DCS, McCann G, Sandhu JK, Quinn PA, McAdam J, Marsh AM, et al. Proenkephalin and prognosis in heart failure with preserved ejection fraction: a GREAT network study. Clin Res Cardiol. 2019;108(8):940-9.

38. Milwid JM, Elman JS, Li M, Shen K, Manrai A, Gabow A, Yarmush J, Jiao Y, Fletcher A, Lee J, et al. Enriched protein screening of human bone marrow mesenchymal stromal cell secretions reveals MFAP5 and PENK as novel IL-10 modulators. Mol Ther. 2014;22(5):999-1007. 
39. Shen B, Harrison-Bernard LM, Fuller AJ, Vanderpool V, Saifudeen Z, El-Dahr SS. The Bradykinin B2 receptor gene is a target of angiotensin II type 1 receptor signaling. J Am Soc Nephrol. 2007;18(4):1140-9.

40. de Oliveira Alvim R, Santos PC, Nascimento RM, Coelho GL, Mill JG, Krieger JE, Pereira AC: BDKRB2 + 9/-9 polymorphism is associated with higher risk for diabetes mellitus in the Brazilian general population. Exp Diabetes Res, 2012, 2012: 480251.

41. Hwangbo C, Wu J, Papangeli I, Adachi T, Sharma B, Park S, Zhao L, Ju H, Go GW, Cui G, et al: Endothelial APLNR regulates tissue fatty acid uptake and is essential for apelin's glucose-lowering effects. Science translational medicine, 2017, 9(407).

42. Lisboa LF, Egli A, Fairbanks J, O'Shea D, Manuel O, Husain S, Kumar D, Humar A. CCL8 and the Immune Control of Cytomegalovirus in Organ Transplant Recipients. Am J Transplant. 2015;15(7):1882-92.

43. Rios-Santos F, Alves-Filho JC, Souto FO, Spiller F, Freitas A, Lotufo CM, Soares MB, Dos Santos RR, Teixeira MM, Cunha FQ. Down-regulation of CXCR2 on neutrophils in severe sepsis is mediated by inducible nitric oxide synthase-derived nitric oxide. Am J Respir Crit Care Med. 2007;175(5):490-7.

44. Zhao Q, Kim T, Pang J, Sun W, Yang X, Wang J, Song Y, Zhang H, Sun H, Rangan V, et al. A novel function of CXCL10 in mediating monocyte production of proinflammatory cytokines. J Leukoc Biol. 2017;102(5):1271-80.

45. Puca AA, Carrizzo A, Spinelli C, Damato A, Ambrosio M, Villa F, Ferrario A, Maciag A, Fornai F, Lenzi P, et al: Single systemic transfer of a human gene associated with exceptional longevity halts the progression of atherosclerosis and inflammation in ApoE knockout mice through a CXCR4-mediated mechanism. Eur Heart J, 2019.

46. Tang Y, He Y, Li C, Mu W, Zou Y, Liu C, Qian S, Zhang F, Pan J, Wang Y, et al: RPS3A positively regulates the mitochondrial function of human periaortic adipose tissue and is associated with coronary artery diseases. Cell Discovery, 2018, 4(1).

47. Hao Y, Kong X, Ruan Y, Gan H, Chen H, Zhang C, Ren S, Gu J. CDK11p46 and RPS8 associate with each other and suppress translation in a synergistic manner. Biochem Biophys Res Commun. 2011;407(1):169-74.

48. Lessard F, Igelmann S, Trahan C, Huot G, Saint-Germain E, Mignacca L, Del TN, Lopes-Paciencia S, Le $\mathrm{CB}$, Montero M. Senescence-associated ribosome biogenesis defects contributes to cell cycle arrest through the Rb pathway. Nat Cell Biol. 2018;20(7):789-99.

49. Palmer ND, Ng MC, Hicks PJ, Mudgal P, Langefeld CD, Freedman BI, Bowden DW. Evaluation of candidate nephropathy susceptibility genes in a genome-wide association study of African American diabetic kidney disease. PLoS One. 2014;9(2):e88273.

50. Wang R, Yoshida K, Toki T, Sawada T, Uechi T, Okuno Y, Sato-Otsubo A, Kudo K, Kamimaki I, Kanezaki $\mathrm{R}$, et al. Loss of function mutations inRPL27andRPS27identified by whole-exome sequencing in Diamond-Blackfan anaemia. Br J Haematol. 2015;168(6):854-64. 
Figures

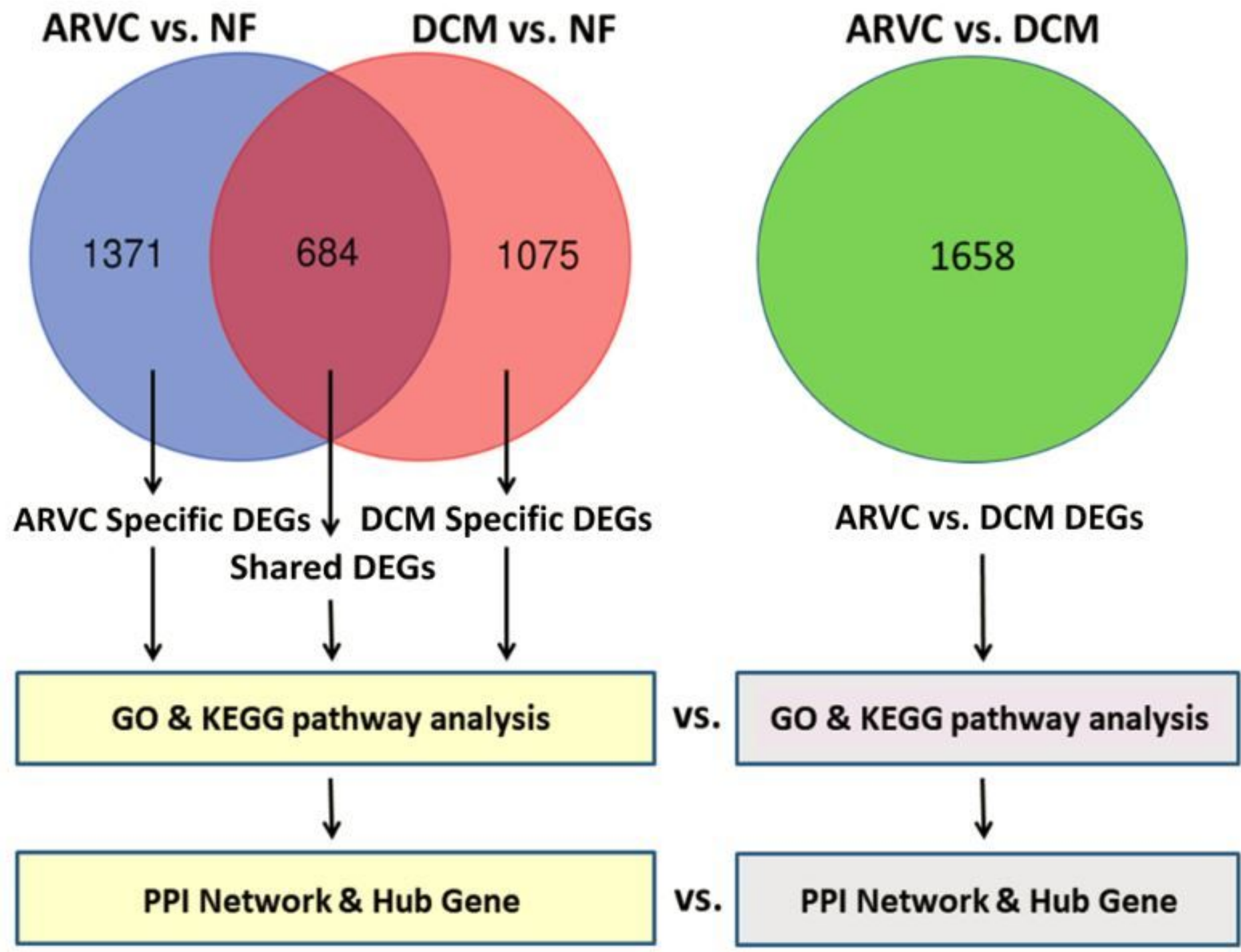

Figure 1

Venn diagram of DEGs of different group and the schematic of DEGs and pathway analysis. Blue circle represents the DEGs of ARVC vs. NF. Pink circle represent the DEGs of DCM vs. NF. Blue-pink part represents the shared DEGs. Green circle represents the DEGs of ARVC vs. DCM. 


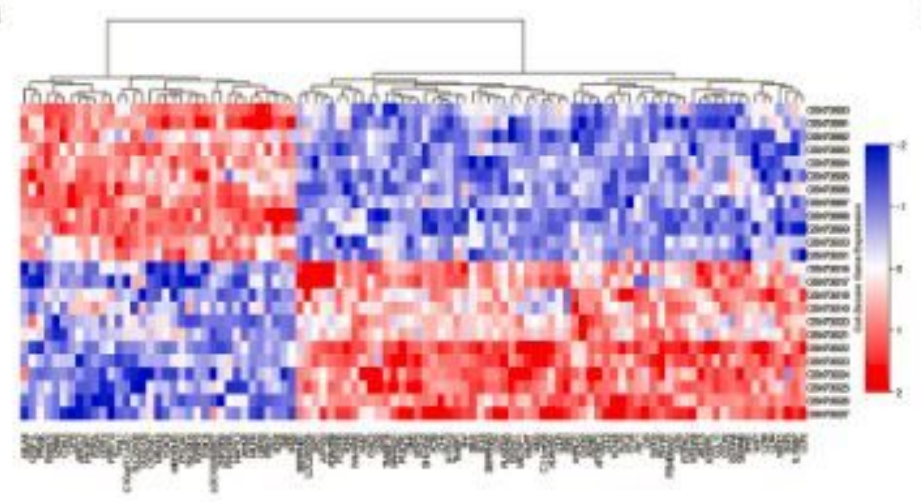

b

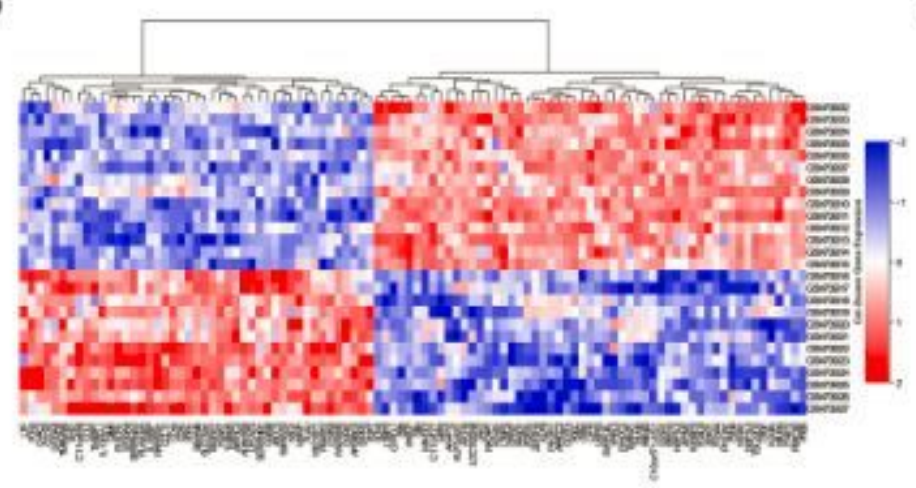

c

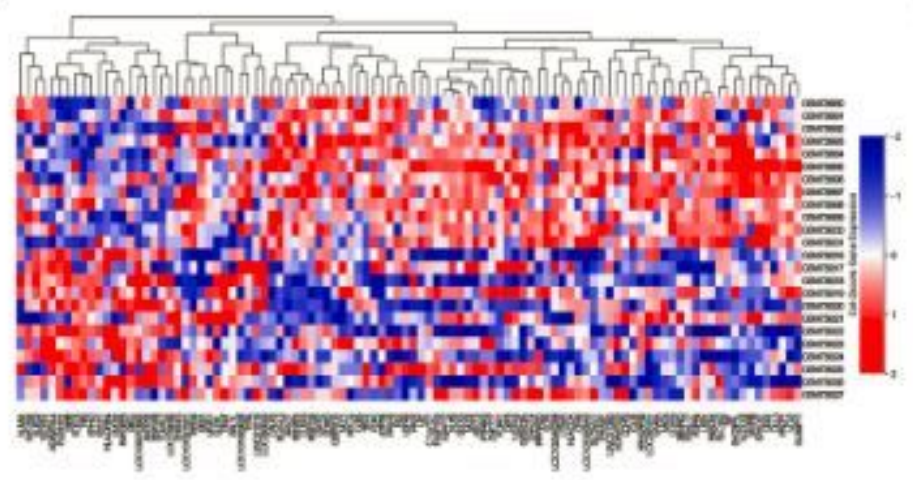

d

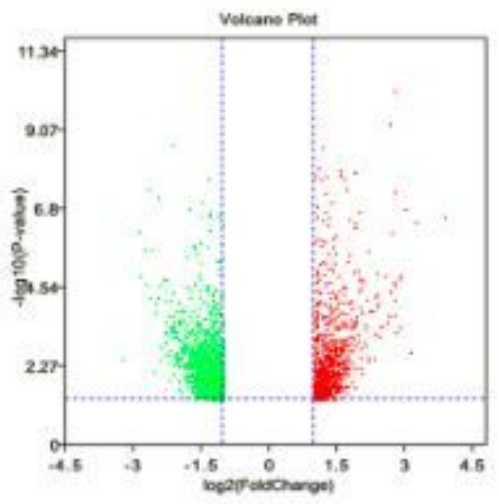

e

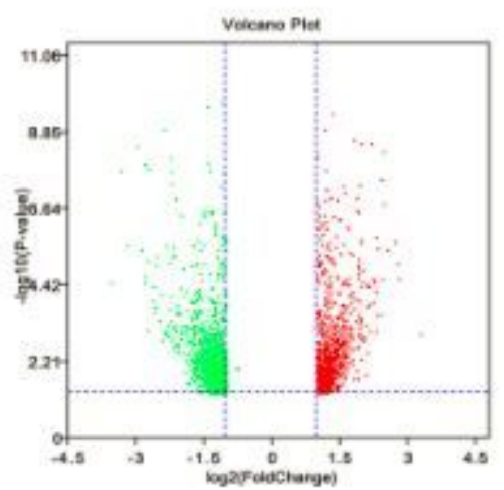

f

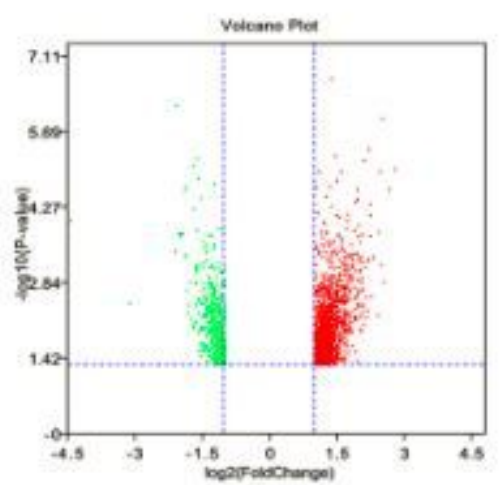

Figure 2

Heat maps and volcano plots of the DEGs. A-C. Heat maps of the top 100 DEGs of ARVC vs. NF, and DCM vs. NF, and ARVC vs. DCM, respectively. Red indicates up-regulated genes and blue indicates downregulated genes. D-F. Volcano plots of the DEGs of ARVC vs. NF, and DCM vs. NF, and ARVC vs. DCM, respectively. The points in volcano plots indicate DEGs with an absolute value of $\| \log 2 \mathrm{FC} \mid>1.0$. Red indicates up-regulated genes and green indicates down-regulated genes. DEGs, differentially expressed genes; FC, fold change. 
a

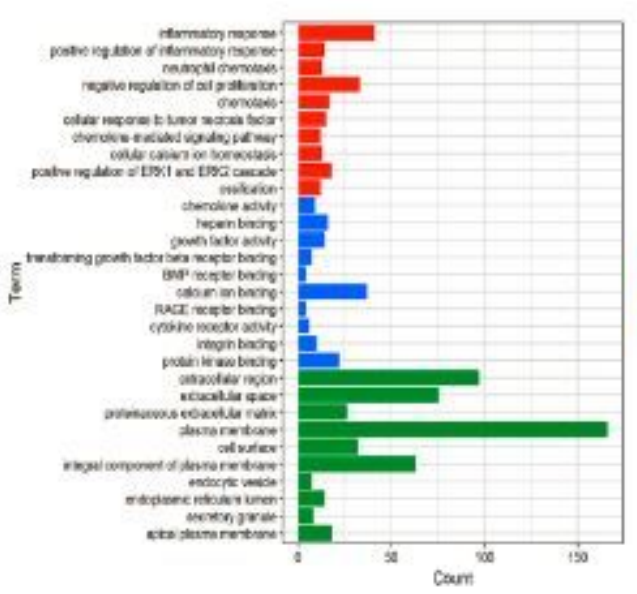

c

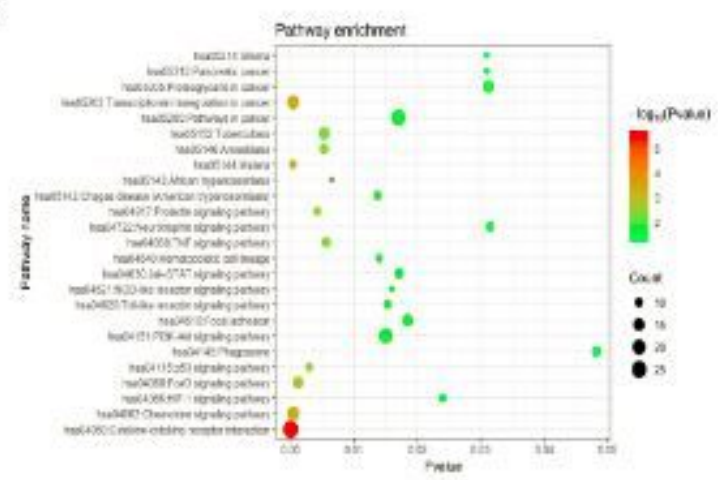

e

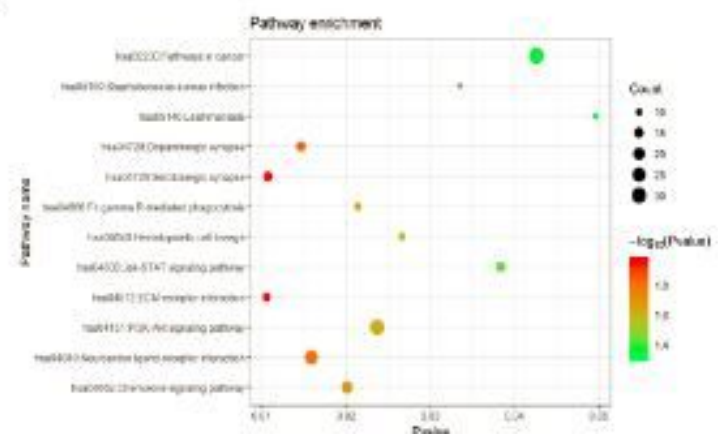

b

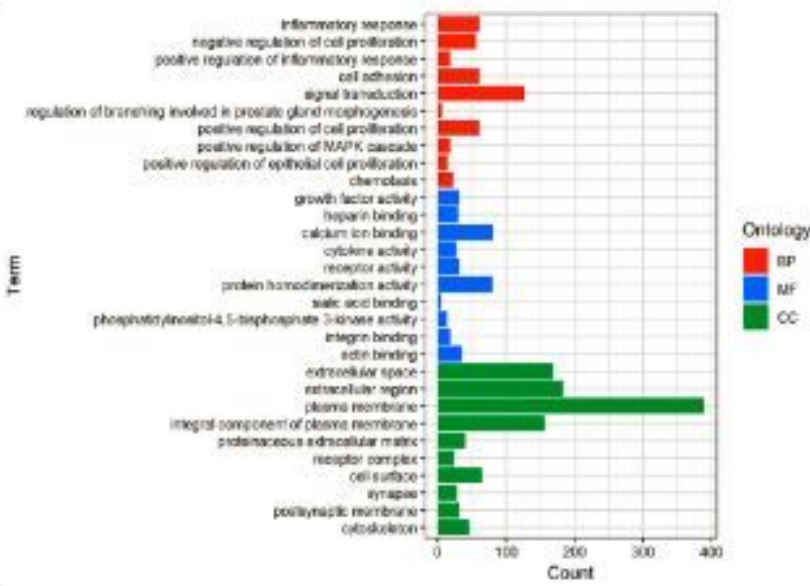

d

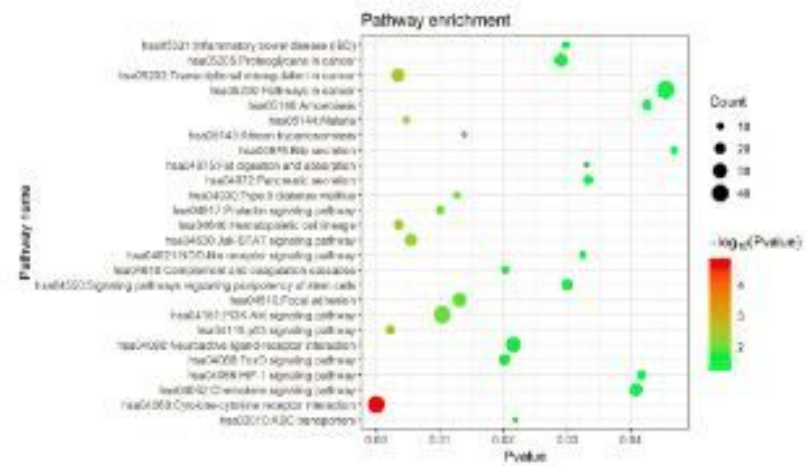

f

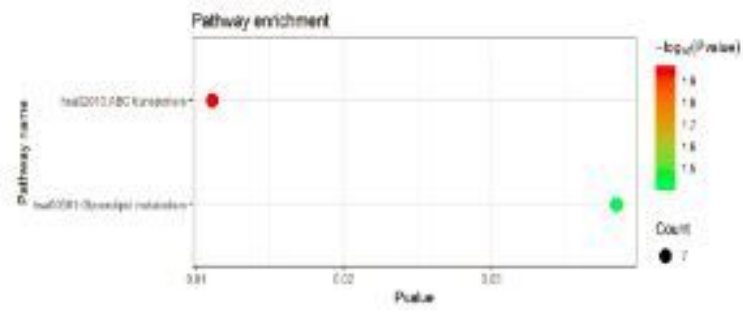

Figure 3

GO and KEGG pathways. A. GO analysis based on the shared DEGs. B. Go analysis based on the DEGs of ARVC vs. DCM. C. KEGG pathways based on the shared DEGs. D. KEGG pathways based on the DEGs of ARVC vs. DCM. E. KEGG pathways based on the special DEGs of ARVC vs. NF. F. KEGG pathways based on the special DEGs of DCM vs. NF. 


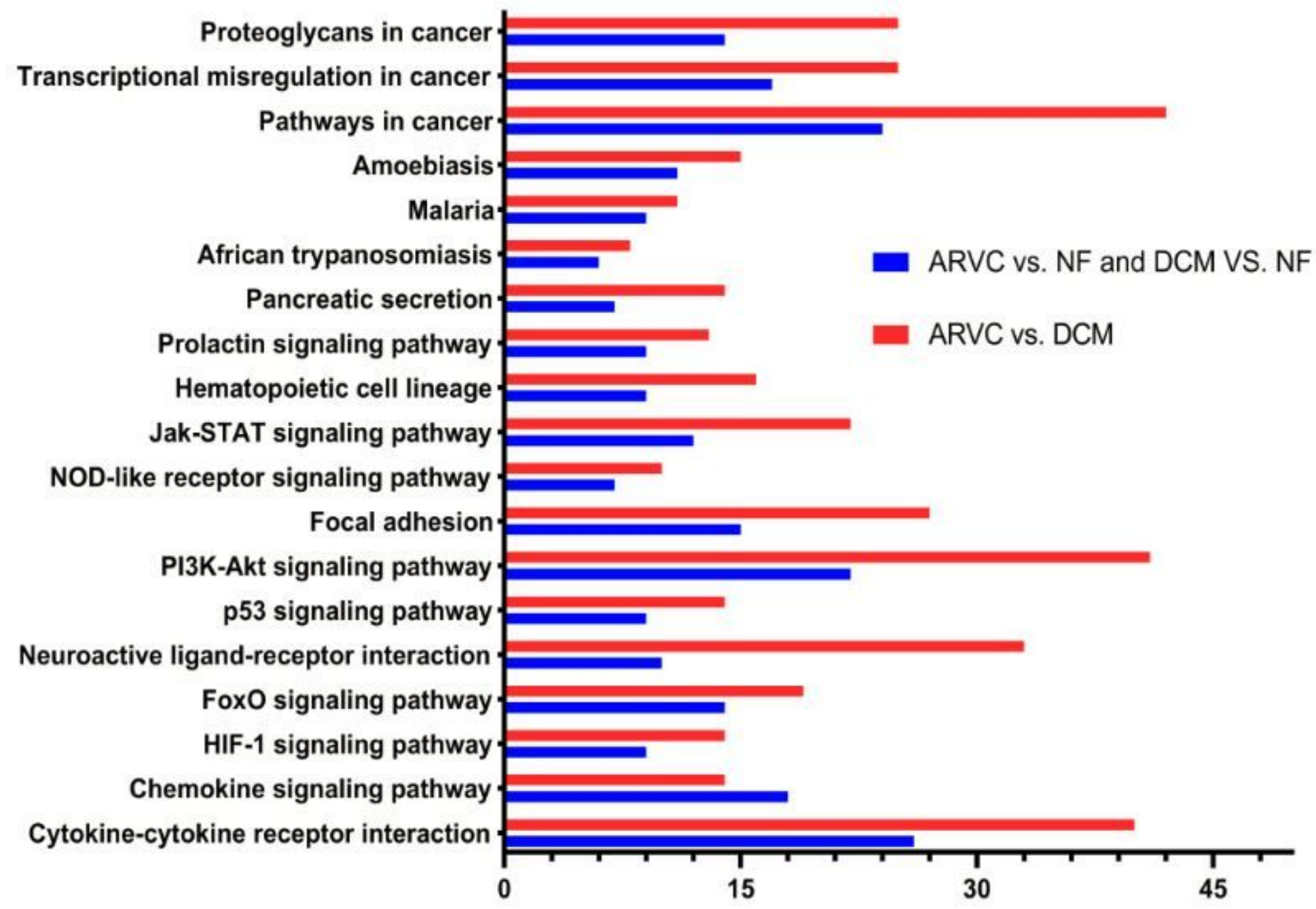

Figure 4

Shared pathways. The blue bars show the shared pathways of the ARVC vs. NF and DCM vs. NF; the red bars show the shared pathways of ARVC vs. DCM. 


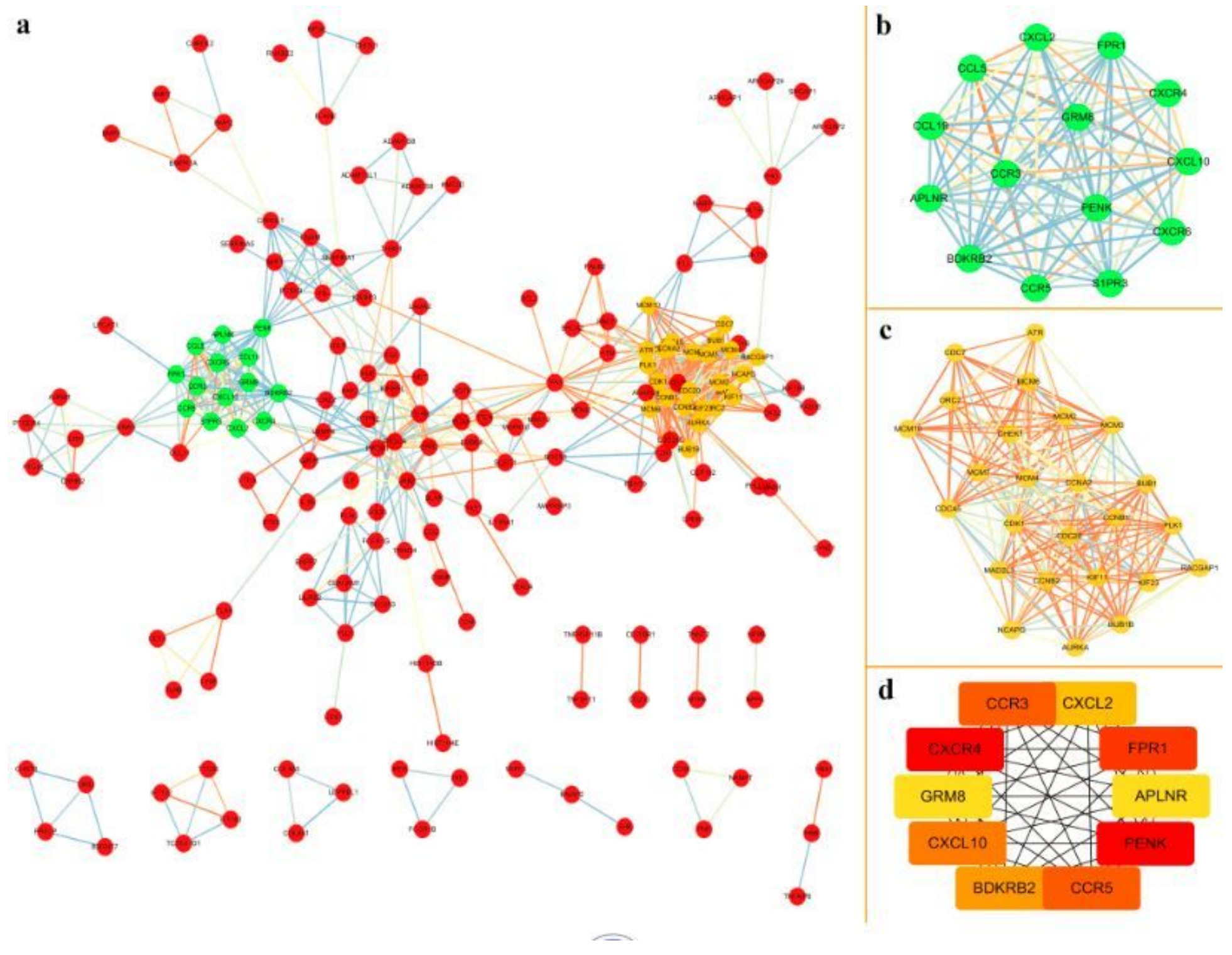

Figure 5

The protein-protein interaction (PPI) network of ARVC vs. NF and DCM vs. NF. A. PPI network. The sequence of the edges' colors is red-orange-blue from high combined score to low combined score. B-C. The modules of the PPI network. Yellow, module 1; green, module 2. D. The top hub genes of the PPI network. The sequence of colors is red-orange-yellow from high ranking to low ranking. 
a
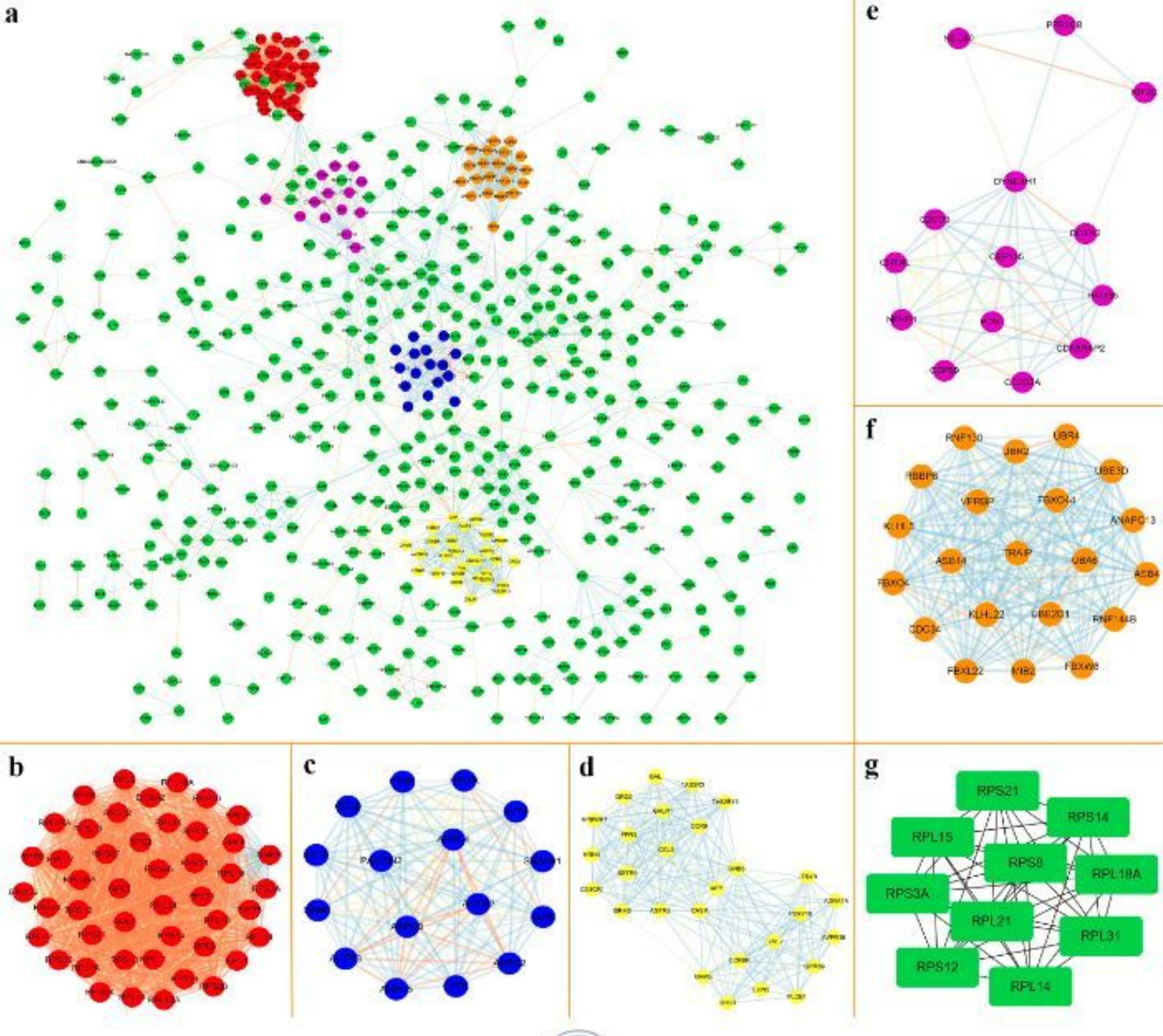

Figure 6

The protein-protein interaction (PPI) network of ARVC vs. DCM. A. PPI network. The sequence of the edges' colors is red-orange-blue from high combined score to low combined score. B-F. The modules of the PPI network. Turquoise, module 1; purple, module 2; dark yellow, module 3; green, module 4; red, module 5. G. The top hub genes of the PPI network. The ranks of the genes are equal.

\section{Supplementary Files}

This is a list of supplementary files associated with this preprint. Click to download.

- Supplymentarytable.docx 